\title{
Artificial Intelligence for Long-term Respiratory Disease Management
}

\author{
Philip A. Catherwood \\ School of Engineering \\ Ulster University \\ Shore Rd., N'abbey \\ Co.Antrim, BT370QB \\ p.catherwood@ulster.ac.uk
}

\author{
Joseph Rafferty \\ Sch. of Computing \& Mathematics \\ Ulster University \\ Shore Rd., N'abbey \\ Co.Antrim, BT370QB \\ j.rafferty@ulster.ac.uk
}

\author{
James McLaughlin \\ School of Engineering \\ Ulster University \\ Shore Rd., N'abbey \\ Co.Antrim, BT370QB \\ jad.mclaughlin@ulster.ac.uk
}

\begin{abstract}
This paper presents the strengths, weaknesses, opportunities, and threats for Artificial Intelligence (AI) as applied to long-term respiratory disease management. This analysis will help to identify, understand, and evaluate key aspects of the technology as well as the various internal/external forces which influence its success in this application space. Such understanding is instrumental to ensure judicial planning and implementation with suitable safeguards being considered. Al has the potential to radically change how respiratory disease management is conducted and may help clinicians to realise new treatment paradigms. The application of Al is clearly not specific to respiratory disease management; however it is a chronic disease that requires on-going monitoring and well evidenced decision making regarding treatment pathways or medication modification. This work emphasises the current position of $\mathrm{Al}$ as applied to respiratory disease management and identifies the issues to help develop strategic directions to ensure successful implementation, evidenced by ubiquitous acceptance and uptake.
\end{abstract}

Artificial Intelligence, Disease management, Machine learning, Respiratory, SWOT

\section{INTRODUCTION}

As medical technology gets smaller, smarter, and more able to process large volumes of data, there are opportunities to take devices which are hospital-based and realise them as a small portable devices, facilitating mobile and remote healthcare strategies. Additionally there is a growing trend to embed intelligence into such devices enabling them to make decisions based on ongoing preferences and inputs, such as biomedical measurements. To this end a software platform, not a clinician, may inform or produce medical decisions about treatment and interventions. Furthermore, emergence of pervasive communications and Internet of Things (IoT) technologies ensure significant data can be relayed to clinical experts and cloud-based software platforms enabling decision support.

There has been a wide range of recent studies where Al has been applied to assist with clinical decisions such as the automated diagnosis of skin cancer [1], bone abnormalities [2], assistance with DNA sequencing [3], and supporting surgery scheduling [4]. Diagnosis of obstructive lung disease is an area of potential promise [5]; it could increase detection accuracy [6] and automate screening to increase patient throughput.
The subject of this recent research has focused around machine learning-based approaches to classification and recognition of activities, notably within the area of Big Data. Generally, these machine learning approaches are within 2 broad categories - supervised and unsupervised. Supervised machine learning builds a classification model through providing it with annotated data, such as images tagged with enumeration representation conditions, such as healthy skin, unhealthy skin, or indeterminate. This model is then applied to previously unseen data to provide a classification.

Unsupervised machine learning leverages unlabelled data to correlate data within a dataset. This enables analysis of data such as matching similar data in groups/clusters or identification of anomalies/outliers. $\mathrm{Yu}$ et al. [1] leveraged supervised machine learning to produce a model which accurately classified the potential for dermoscopy images to be cancerous, Deep Convolutional Neural Networks (CNN) were used to realise this solution producing a viable trained model from 900 images. Jagadev et al. [2] implemented supervised machine learning to produce a Support Vector Machine-based classifier capable of identifying images of blood smear tests which are likely to have a form of leukaemia. 
Celesti et al. [3] surveyed studies which involved Al-based analysis of genomics data to identify conditions and anomalies. These surveyed studies used supervised and unsupervised machine learning as dictated by their use case. Typically, supervised learning was employed to identify conditions using a model built from training data whereas unsupervised machine learning was used to identify anomalies.

In recent years $\mathrm{Al}$ research has had a focus on using machine learning, however, works exist which have devised Decision Support Systems (DSS) support systems and traditional, tailored, classification algorithms. Xiang et al. [4] have produced a DSS incorporating custom classification algorithms to optimise scheduling of surgical procedures giving operational restraints and required resources, such as availability of an operation theatre and supporting staff. This has proven to optimise efficiency and increase health outcomes.

All illness management may benefit from $\mathrm{Al}$; respiratory disease management is an enduring key medical theme which encompasses Asthma, chronic obstructive pulmonary disease (COPD), pulmonary hypertension, bronchiectasis, sleep apnoea, etc. [7] and attributes to around $20 \%$ of UK deaths [8]. COPD is ranked as the third most common global cause of death in the last decade [9].

Respiratory disease is commonly diagnosed and monitored using Spirometry (lung function test) which evaluates the volume of air expelled from the lungs (forced vital capacity) as well as the rate at which the air is expelled (forced expiratory volume) [10]. A similar, simpler, meter measuring the peak expiratory flow (PEF) is often used in everyday clinical practice (often referred to as peak flow meter). This technology has been mechanical in nature but in recent years has been realised as electronic measurement devices with wireless connectivity, typically Bluetooth. Other diagnosis techniques which complement spirometry include the use of biomarkers for COPD or lung cancer [11, 12]. Such biomarkers may be realised as implantable monitors or home test kits; employing Al would make a powerful point-of-care system.

There are clear correlations between air pollution and respiratory disease, mortalities, and outpatient admissions [13]. Approximately 3 million people die each year from COPD, which is estimated to be around $6 \%$ of all deaths worldwide. It is also determined that some 235 million people suffer from asthma [7]. While significant progress has been made to predict, monitor and treat respiratory disease [14-16] there is still significant exploration required.
Given the types of data and availability of datasets a swathe of $\mathrm{Al}$ techniques can be used within this domain. Supervised machine learning may be used to build a classification model which may be used to indicate when COPD symptoms are likely to deteriorate. This model may be produced from an annotated dataset leveraging environmental metrics, biomarkers, an individual's medical history and current state. Unsupervised machine learning may be applied to a large dataset gathered from sufferers to help identify factors which can contribute to, or prevent, COPD flare ups.

A DSS may be produced from domain knowledge to inform, prompt, and advise respiratory disease suffers in a tailored fashion. This DSS may take inputs from PEF measurements, environmental data, and supervised machine learning classification of an individual's parameters. The realisation of such a solution will be the subject of future work and will be informed by the quality and quantity of data that can be reliably obtained from sufferers of respiratory diseases.

\section{S.W.O.T. ANALYSIS}

An analysis of the strengths, weaknesses, opportunities, and threats of Al's application to respiratory disease management is an essential aspect of understanding the potential of the technology as well as the paths to application. The strengths and weaknesses are with respect to the internal aspects of Al. Any identified weaknesses may be addressed via technological revisions. The opportunities and threats are with respect to the external aspects; these include other technologies, public perception of the technology, financial climate, etc. Threats are addressed via methods such as legislation and public education strategies, although are more difficult to change. The use of SWOT analysis gives a panoramic and comprehensive perspective of the state of the technology in the specific sector. It is a universal method which creates a usable examination which may act as a starting point to tackle the challenges.

\subsection{Strengths}

Al boasts the ability to recognise objects in images, transcribe speech, control machines, analyse DNA to detect genomic conditions, etc. [17]. Such technological strengths expedite the delivery of bespoke personalised care for respiratory disease management. Al's ability to learn, find patterns, make decisions, etc. This may be facilitated through sensor measurements from spirometers, inhaler and medication usage, biomarkers, etc. In addition, qualitative data may be incorporated from active assistants/chat bots to understand feelings, pain, anxiety, breathlessness, etc. Such solutions will assist with making informed machine decisions 
leading to medication alteration, clinician intervention or hospitalisation. Al could predict a respiratory disease decline, such as an asthma attack, before the patient even considers there to be anything untoward or peak flow measurements show a notable decline in lung function. The abundant recorded data that can be stored from the vast array of patients can help the training of accurate Al models, particularly through deep learning approaches. It is recognised that early detection of exacerbations in COPD can increase positive outcomes and reduce hospital admissions; telehealth-based systems interventions can decrease the costs associated with COPD patients [18] and promote better self-management [19, 20].

\subsection{Weaknesses}

In late 2017 mainstream technical news sources published reports with taglines such as "No one really knows how the most advanced algorithms do what they do" [21]. This suggests that the decisions that Al makes can be unexplainable or beyond logic. For example, chip maker Nvidia tested a car controlled by $\mathrm{Al}$ and later reported that the vehicle didn't follow a single instruction provided by the programmers, instead using an algorithm that it had taught itself. While this may be good for attentiongrabbing headlines it creates disquiet for those who are looking to $\mathrm{Al}$ to intelligently manage the health decisions of the chronically ill. The vehicle in the test conducted itself appropriately and completed the task very well but the engineers couldn't describe how it did it [21]. This is not an isolated report but a growing trend [22]. Furthermore, some complex Al systems have been described as "inscrutable" as it is not possible to make the Al system explain why it made certain decisions; such inability for humans to understand the decisionmaking processes of the system creates significant risk. These inscrutable solutions typically leverage machine learning based approaches to Al. When that risk is applied to chronic diseases like COPD such inscrutable systems are unacceptable and it would not be possible to verify if a death was an unavoidable outcome or a bizarre Al decision. Validation of medical technology requires substantial clinical testing which costs time and money; however system reliability and observability are two of the key parameters of any system under test. Al in healthcare cannot be a leap of faith into the unknown and capricious systems may have no future in this space. To this end, such solutions may be limited to offer insight to inform decisions to be made by medical practitioners.

\subsection{Opportunities}

With respiratory disease being a significant strain on healthcare resources it is advantageous to push the monitoring of patients into the community to keep patients out of hospital [18]. Smart homes are increasing popular [23] and there may be opportunities to integrate medical devices with these networks. Implementing smart healthcare devices into the community also helps to drive technology and services into remote areas to address the recognised issues of rural communities being last to get the latest technical services [24]. Having intelligent sensors and systems that are continually monitoring your wellness is likely to have measurable health benefits over occasional clinic visits; such monitoring resolution will assist with faster responses to deteriorations as well as better diagnoses. This principle has been proven already with smart implanted insulin pumps which actively monitor blood glucose levels and automatically medicate to exacting levels accordingly [25]. Further emerging technologies such as stretchable electronics [26] and long range low power communication systems [27] increase the potential for effective technology deployment.

\subsection{Threats}

The literature shows limited work on $\mathrm{Al}$ and telemetry for respiratory disease monitoring [28] which means it is not as well understood as some other areas of study. However, some key explorations on the use of machine learning for respiratory disease diagnosis in clinical settings serve to effectively highlight the value of developing the technology [29-31]. Other external threats may impede the uptake of Al for respiratory disease management including the fear of lawsuits against the system creator if $\mathrm{Al}$ is proven to have made a poor judgement or an obvious mistake. Likewise, the over-expectation of investors, technical communities and medical professionals, fears over security of data and hacking, poor investment into the technology, user error of any aspect of the system, issues with poor battery performance for portable devices, and global economics which demands continual cost cutting will all serve to threaten successful adoption of the technology. High profile figures such as the late Professor Stephen Hawking have done little to help the public perception of Al [32]. Furthermore the notoriously slow uptake of new technology in healthcare [33] may result in the technology being quickly outdated, especially in the context of Al technology changing so rapidly.

A notable high profile Al failure will no doubt cast a long shadow over the trust of Al implementation for some time; in March 2018 an autonomous car being driven by $\mathrm{Al}$ was involved in a fatal pedestrian accident [34]. Internet commentators reasoned that the outcome would have been the same regardless of whether a computer or a person was under control of the vehicle, nonetheless the headline will still capture the imagination of those already fearful of machines 
making life and death decisions. These issues are real and cannot be overcome easily; objections will remain and as such the technology could struggle to enjoy widespread acceptance without significant research and validation of medical Al systems. While automotive Al applications are clearly distinct from healthcare applications the public perception may not be adequately informed to appreciate the different techniques and technologies utilised.

\section{CONCLUSIONS}

The potential for impact and reduced costs for respiratory disease management is clear, however there are also significant obstacles to implementation and the approval processes in healthcare could make the new technology lag behind $\mathrm{Al}$ implementation in other sectors; healthcare could therefore be the last sector to experience the benefits of the IOT/AI revolution. Correct implementation however will fundamentally enable fast reactions to emergencies in the short term and disease progression management in the long term. It will also have the capacity to address the increased management complications presented by multiple chronic conditions (MCC).

Non-medical devices purporting to contain Al may serve to impede authorised application in clinical practice as if these consumer electronics lifestyle devices are of questionable trustworthiness they may complicate acceptance of $\mathrm{Al}$ in automated healthcare decision making. General recommendations for well-managed implementation includes ensuring the technology is not hurried to meet industry expectations, the use of valid training datasets taken from a cross section of communities and diseases, ongoing healthcare cost evaluations, and the acknowledgement that $\mathrm{Al}$ is still in its infancy and has significant maturing to do before being ready for such an audacious task.

\section{REFERENCES}

[1] Yu, L., Chen, H., Dou, Q., Qin J., Heng, P.A. (2017) Automated Melanoma Recognition in Dermoscopy Images via Very Deep Residual Networks. IEEE T. Med. Im., vol. 36, pp. 994-1004.

[2] Jagadev P., Virani, H.G. (2017) Detection of leukemia and its types using image processing and machine learning, Intl. Conf. Trends Elect. Info. (ICEI), Tirunelveli, pp. 522-526. IEEE, USA.

[3] Celesti, F., et al., (2017) Big data analytics in genomics: The point on Deep Learning solutions. IEEE Symp. Comp. Comms. (ISCC), Heraklion, pp. 306-309. IEEE, USA.
[4] Xiang, W., Yin, J., Lim, G. (2015) A short-term operating room surgery scheduling problem integrating multiple nurses roster constraints. Art. Int. in Med., vol. 63, pp. 91-106.

[5] Das, N., Topalovic, M., Janssens, W. (2018) Artificial intelligence in diagnosis of obstructive lung disease: current status and future potential. Curr. Opin. Pulm. Med. vol. 24, pp. 117-123.

[6] IEEE Spectrum News. (2017) Al versus doctors. spectrum.ieee.org/static/ai-vs-doctors (6/3/18).

[7] British Lung Foundation (2018) Lung disease in the UK. https://statistics.blf.org.uk/lung-disease-ukbig-picture $(5 / 6 / 18)$

[8] WHO. (2018) Chronic respiratory diseases. http://www.who.int/respiratory/en/ (6/3/18).

[9] Patel, J., et al. (2014) Global and regional trends in mortality from chronic obstructive pulmonary disease: their relation to poverty, smoking and population change. Eur. Respir. Journal. vol. 44, pp. 1239-1247.

[10] Komarow, H.D., et al. (2011) Impulse oscillometry in the evaluation of diseases of the airways in children. Ann. Allergy Asthma Immunol., vol. 106, pp. 191-199.

[11] Khan, K., et al. (2017) Potential biomarkers for the diagnosis of respiratory tract infection and lungs cancer. Cell Molecular Biology. vol. 30, pp. 46-52.

[12] Kim, H.J., Choi, M.G., Park, M.K., Seo, Y.S. (2017) Predictive and Prognostic Biomarkers of Respiratory Diseases due to Particulate Matter Exposure. J. Cancer Prev. vol. 22, pp. 6-15.

[13] Mo, Z., et al. (2018) Acute effects of air pollution on respiratory disease mortalities and outpatients in Southeastern China. Nature. vol. 8, pp. 1-9. Springer, USA.

[14] Giannaccini, M.E., et al. (2017) Respiratory simulator for robotic respiratory tract treatments. IEEE Intl. Conf. Robotics and Biomimetics, Macau, Macao, 5-8 Dec. 2017, pp. 2314-2319, IEEE, USA..

[15] Rehouma, H., Noumeir, R., Jouvet, P., Bouachir, W., Essouri, S. (2017) A computer vision method for respiratory monitoring in intensive care environment using RGB-D cameras. $7^{\text {th }}$ Intl. Conf. Im. Proc. Theory, Tools and App., Montreal, QC, 28th Nov. $-1^{\text {st }}$ Dec. 2017, pp. 1-6, IEEE, USA.

[16] Nousias S., et al., (2018) An mHealth System for Monitoring Medication Adherence in Obstructive Respiratory Diseases Using Content Based Audio Classification. IEEE Access, vol. 6, pp. 1187111882. 
[17] Newton-Rex, E. (2017) Business Insider. http://uk.businessinsider.com/artificial-intelligence-aimost-impressive-achievements-2017-

3 ? $r=U S \& I R=T / \#$ what-ai-cando-everyday-humanstuff- 1 $(24 / 3 / 18)$.

[18] Guarascio, A.J., Ray, S.M., Finch, C.K. (2013) The clinical and economic burden of chronic obstructive pulmonary disease in the USA. Clinicoecon Outcomes Res. vol. 5, pp. 235-245.

[19] Fernandez-Granero, M.A., Sanchez-Morillo D., Leon-Jimenez A. (2018) An artificial intelligence approach to early predict symptom-based exacerbations of COPD. Biotech. \& Biotech. Equipment, DOI: 10.1080/13102818.2018.1437568

[20] Esteban, C., et al. (2015) Machine learning for COPD exacerbation prediction. Eur. Resp. J. vol. 46: OA3282.

[21] Knight, W. (2017) MIT. www.technologyreview.com/s/604087/the-darksecret-at-the-heart-of-ai/ (3/3/18)

[22] Gershgorn, D. (2017) Quartz, qz.com/1146753/ai-is-now-so-complex-its-creatorscant-trust-why-it-makes-decisions/ (3/3/18)

[23] Wetzel, K. (2018) Digital Trends www.digitaltrends.com/home/smart-home-technologytrends-and-whats-ahead/ (21/3/18).

[24] Ofcom(2017) Ofcom www.ofcom.org.uk/aboutofcom/latest/media/media-releases/2017/northernireland-broadband $(21 / 3 / 18)$

[25] Pickup, J., Holloway, M., Samsi, K. (2015) Real-time continuous glucose monitoring in type 1 diabetes: a qualitative framework analysis of patient narratives. Dia. Care. vol. 38, pp. 544-550.

[26] Son, D., et al, (2014) Multifunctional wearable devices for diagnosis and therapy of movement disorders. Nature Nanotech. vol. 9, pp. 397-404.

[27] Catherwood, P., McComb, S., Little, M., McLaughlin, J, (2017). Channel Characterisation for Wearable LoRaWAN Monitors, Loughborough Ant. and Prop. Conf. (LAPC), 12-13 Nov. 2017, pp. 1-4. IET, UK.

[28] Sanchez-Morillo, D., Fernandez-Granero, M.A., Leon-Jimenez, A. (2016) Use of predictive algorithms in-home monitoring of chronic obstructive pulmonary disease and asthma. Chronic Resp. Disease. vol. 13, pp. 264-283.
[29] Prasad B., Prasad P.K., Sagar, Y. (2011) Advances in computer science and information technology. Springer, Berlin, pp. 570-576.

[30] Pescatore A.M., et al. (2014) A simple asthma prediction tool for preschool children with wheeze or cough. J All. Clin. Immun. vol. 133, pp. 111-118.

[31] Badnjevic A, et al. (2015) Neuro-fuzzy classification of asthma and chronic obstructive pulmonary disease. BMC Med. Inform. Decision Making. vol. 15, pp. S1.

[32] Cellan-Jones, R. (2014) BBC. www.bbc.co.uk/news/technology-30290540 (19/2/18)

[33] Palabindala, V., Pamarthy, A., Jonnalagadda, N.R. (2016) Adoption of electronic health records and barriers. J. Community Hosp. Intern. Med. Perspect. vol. 6, DOI: 10.3402/jchimp.v6.32643

[34] Wakabayashimarch, D. (2018) NY Times. https://www.nytimes.com/2018/03/19/technology/uber -driverless-fatality.html (29/3/18). 